\title{
LA DÉRIVÉE FRACTIONNAIRE EN RHÉOLOGIE DES POLYMĖRES - APPLICATION AUX COMPORTEMENTS ÉLASTIQUES ET VISCOÉLASTIQUES LINÉAIRES ET NON LINÉAIRES DES ÉLASTOMÈRES
}

\author{
M. SOULA ET Y. CHEVALIER
}

\begin{abstract}
RÉsumÉ. Les applications des méthodes basées sur les systèmes différentiels fractionnaires deviennent de plus en plus fréquentes dans de nombreux domaines scientifiques et en particulier dans le domaine de la rhéologie expérimentale des solides.

Dans cet article, nous appliquons une méthode fondée sur les systèmes différentiels fractionnaires pour modéliser le comportement mécanique des polymères et élastomères. Il s'agit d'une approche paramétrique ajustée sur des résultats expérimentaux afin de modéliser le module d'Young complexe de matériaux viscoélastiques en fonction de la fréquence. Cette utilisation de la dérivée fractionnaire, économique en paramètres, permet de modéliser le matériau dans une large gamme de fréquences. Elle autorise en outre un passage au régime temporel par transformée de Fourier inverse et permet également l'obtention des fonctions de fluage et de relaxation, renseignements utiles dans l'étude des phénomènes transitoires lents.

Cette démarche est enfin extrêmement utile dans l'analyse de la viscoélasticité non linéaire (vibrations sous précharge), domaine où les renseignements expérimentaux sont extrêmement réduits.
\end{abstract}

\begin{abstract}
Applications of fractional dérivatives are extensively used in many scientific fields and especially in the field of experimental rheology of solids.

In this paper models of polymeric and rubber materials are made of fractional derivatives. Complex Young moduli of viscoelastic materials versus frequency are fitted on experimental data. The fractional derivative is efficient to obtain, over a large range of frequencies, closed form expression of Young modulus. Inverse Fourier transform provides creep and relaxation function which are significant in slow transient motions.

Because of the small amount of data, fractional derivative is a useful tool to analyse non linear behavior of viscoelastic materials.
\end{abstract}

\section{INTRODUCTION}

Dans la formulation des lois de comportement thermomécaniques des polymères et élastomères deux approches sont traditionnellement utilisées. La première, qui est une description non paramétrique du phénomène, présente l'avantage de s'affranchir de la modélisation mais réduit l'information à un intervalle de temps (ou de fréquences) de faible amplitude. La deuxième approche est une modélisation paramétrique qui fournit, après ajustement des paramètres sur les données expérimentales, une connaissance du comportement rhéologique du matériau dans une très large gamme de fréquences (ou de temps). Le choix de la modélisation reste ouvert. L'utilisation de la dérivée fractionnaire répond à un souci de réduction du

(C) Société de Mathématiques Appliquées et Industrielles. Typeset by LATEX.

M. Soula : IPEI de Mateur -7030 Mateur (Tunisie).

Y. Chevalier : ISMCM, LISMMA Groupe rhéologie et strucures, 3, Rue Fernand Hainaut 93407 Saint Ouen, France. Email : chevalie@ismcm-cesti.fr. 
nombre de paramètres de la loi de comportement mécanique du matériau: quatre ou cinq au plus au lieu d'une dizaine par les opérateurs différentiels classiques.

Les élastomères travaillent fréquemment sous précharge: petites déformations dynamiques autour d'une déformation statique importante (Cas des silent blocs par exemple). L'analyse du comportement se fait alors dans le cadre de la viscoélasticité non linéaire. Bien que le problème de modélisation générale reste encore ouvert, la dérivée fractionnaire permet d'exploiter efficacement les résultats expérimentaux réduits, et ceci dans un contexte industriel.

Depuis plusieurs décennies, de nombreux auteurs [11], [19], [5], [13], [12], [7] ont utilisé des modéles rhéologiques (donc paramétriques) basés sur des arrangements en série ou en parallèle de cellules élémentaires modélisées par des dérivées fractionnaires, ces dernières remplaçant les traditionnels ressorts et amortisseurs. La dérivée fractionnaire peut en effet être interprétée mécaniquement comme le passage continu de l'état de ressort (exposant nul) à celui d'amortisseur (exposant égal à 1) comme l'a indiqué T. Vinh en 1967 [22] lors d'une étude de quelques modèles basés sur les dérivées fractionnaires.

Depuis les années 80 cette formulation a intéressé un grand nombre de mécaniciens et de rhéologues, citons Bagley et Torvik [3], [2] par exemple.

\subsection{Formulation mathématique}

On doit à Leibniz (1665) [9] l'idée des dérivées fractionnaire (ou d'ordre non entier). La première grande théorie de la dérivation fractionnaire est due à Louville (1832), mais la formule la plus souvent utilisée aujourd'hui, dite intégrale de Riemann-Liouville, fut donnée par Riemann (1847). Sur ces aspects historiques, on pourra se référer aux travaux de $\mathrm{S}$. Dugowson [6].

L'opérateur de dérivée fractionnaires d'une fonction temporelle: $D^{\alpha} f(t)$, peut être défini par deux méthodes. Bien que ces deux définitions soient équivalentes, la deuxième convient mieux dans les programmes de calcul (en éléments finis par exemple).

1.1.1. Première DÉfinition DÉcoulant DES TRANSFormées intégrales de LAPLACE. Dans cette première définition, la dérivée d'ordre $\alpha<0$ d'une fonction causale $f(t)$ est donnée par l'intégrale de Riemann-Liouville:

$$
D^{\alpha} f(t)=\int_{0}^{t} \frac{\xi^{-\alpha-1}}{\Gamma(-\alpha)} f(t-\xi) d \xi .
$$

Lorsque $\alpha>0$, la dérivée $D^{\alpha} f(t)$ est définie par dérivations aux ordres entiers de la formule ci-dessus. De manière équivalente, on peut poser :

$$
D^{\alpha} f(t)=\mathrm{PF} \int_{0}^{t} \frac{\xi^{-\alpha-1}}{\Gamma(-\alpha)} f(t-\xi) d \xi, \alpha>0, \alpha \notin \mathbf{N}
$$

où PF représente la partie finie de l'intégrale (L. Schwartz), $\Gamma$ est la fonction factorielle (ou Gamma) telle que:

$$
\Gamma(a)=\int_{0}^{\infty} x^{a-1} e^{-x} d x, \Re(a)>0 .
$$

Remarque 1.1. on peut calculer $\Gamma(a)$ pour $a<0$ par cette intégrale et par prolongement analytique avec la condition $a=\alpha$ non entier.

1.1.2. Deuxième DÉfinition À PARTiR DE LA DÉrivée D'ordre Entier. Une deuxième définition de la dérivée fractionnaire d'une fonction $f(t)$ fondée sur les différences finies, à été donnée par J. Liouville [10], A. K. Grünwald [8]. Elle équivaut à la précédente en passant à la limite quand le pas de discrétisation $h$ tend vers 
0 , dans le cas où $f$ est causale, tandis que dans le cas général elle correspond au choix, dans l'intégrale de Riemann-Liouville, d'une borne inférieure égale à $-\infty$.

Une définition plus générale que la précédente peut être obtenue à partir de la définition classique de la dérivée d'ordre entier réel (J. Liouville [10], A. K. Grünwald $[8])$ :

$$
D^{\alpha} f(t)=\sum_{k=0}^{\infty}(-1)^{k}\left(\begin{array}{c}
\alpha \\
k
\end{array}\right) f(t-k h) .
$$

On peut se faire une idée de l'équivalence de ces deux définitions en utilisant la définition donnée à la fonction factorielle (ou Gamma) $\Gamma(\alpha)$ par Gauss, soit:

$$
\Gamma(\alpha)=\lim _{k \rightarrow \infty} \frac{k ! k^{\alpha}}{\alpha(\alpha+1) \ldots(\alpha+k)} .
$$

La dérivée fractionnaire d'une fonction à un instant $t$ donné prend en compte les valeurs de cette fonction à tous les instants du passé, elle donne une caractérisation globale de la fonction $f$.

\subsection{Domaines D'application}

Les applications de la méthode à dérivation fractionnaire dans les sciences physiques et les sciences de l'ingénieur relèvent des contributions scientifiques de ces dernières décennies. Cette méthode est utilisée comme outil de modélisation dans plusieurs domaines [14].

En mécanique et en rhéologie, l'application de la méthode à dérivation fractionnaire pour modéliser le comportement des matériaux trouve une base théorique dans la théorie microstructurale de Rouse [12] d'une part et elle s'appuie sur les lois de la thermodynamique, Bagley et Torvik [3] d'autre part.

\section{LOI DE COMPORTEMENT À BASE DES DÉRIVÉES FRACTIONNAIRES EN RÉGIME HARMONIQUE}

La loi de comportement à base de dérivées fractionnaires liant les contraintes aux déformations pour un matériau viscoélastique en régime harmonique a été proposée par Vinh [22] (1967). Si nous prenons l'expression la plus simple en dérivées non entières, le module d'Young complexe $E^{*}(\omega)$ est donné, en fonction de $\omega$, par la forme suivante [22] :

$$
E^{*}(\omega)=\frac{\sigma^{*}(\omega)}{\varepsilon^{*}(\omega)}=E_{0} \frac{\prod_{i=1}^{n}\left(1+\left(j T_{i} \omega\right)^{\alpha_{i}}\right)}{\prod_{k=1}^{q}\left(1+\left(j T_{k} \omega\right)^{\alpha_{k}}\right)}
$$

dans laquelle $T_{i}$ est une constante homogène à un temps.

Si nous ne prenons que les premiers termes de la relation (2.1), le module d'Young complexe peut prendre la forme suivante:

$$
E^{*}(\omega)=E_{0} \frac{1+\left(j T_{1} \omega\right)^{\alpha_{1}}}{1+\left(j T_{2} \omega\right)^{\alpha_{1}}}
$$

c'est l'écriture la plus simple que l'on peut utiliser pour un matériau représenté par 4 paramètres. Dans (7) les exposants du numérateur et du dénominateur sont égaux (ici $\alpha_{1}$ ) pour satisfaire la condition de comportement asymptotique des solides lorsque la pulsation $\omega$ tend vers l'infini. En effet on doit obtenir un module complexe fini :

$$
E_{\infty}=E_{0}\left(j T_{1} \omega\right)^{\alpha_{1}} /\left(j T_{2} \omega\right)^{\alpha_{1}} \text { quand } \omega \rightarrow \infty .
$$


Remarque 2.1. Le cas où les exposants au numérateur et au dénominateur sont différents correspond au comportement d'un fluide.

La loi de comportement liant la contrainte complexe $\sigma^{*}$ à la déformation complexe $\varepsilon^{*}$ prend alors la forme suivante:

$$
\left(1+\left(j T_{1} \omega\right)^{\alpha_{1}}\right) \sigma^{*}(\omega)=E_{0}\left(1+\left(j T_{2} \omega\right)^{\alpha_{1}}\right) \varepsilon^{*}(\omega) .
$$

Une deuxième forme de la loi de comportement à base des dérivées fractionnaires à trois dimensions liant les contraintes aux déformations pour un matériau viscoélastique homogène et isotrope a été proposée par Bagley et Torvik [3] et [2] (1984). Cette loi prend la forme suivante:

$$
\begin{gathered}
\left(1+\sum_{k=1}^{K} a_{k} D^{\beta_{k}}\right)\left(1+\sum_{p=1}^{P} b_{p} D^{\beta_{p}}\right) \sigma_{m n}(t)= \\
\delta_{m n}\left(1+\sum_{p=1}^{P} b_{p} D^{\beta_{p}}\right)\left(\lambda_{0}+\sum_{j=1}^{J} \lambda_{j} D^{\alpha_{j}}\right) \sum_{k} \varepsilon_{k k}(t) \\
+2\left(1+\sum_{k=1}^{K} a_{k} D^{\beta_{k}}\right)\left(\mu_{0}+\sum_{l=1}^{L} \mu_{j} D^{\alpha_{l}}\right) \varepsilon_{m n}(t),
\end{gathered}
$$

où $\delta_{m n}$ est le symbole de Kronecker. On assemble ainsi dans (2.4) les composantes isotropes et déviatoires des tenseurs de contraintes et de déformations. $\sigma_{m n}$ et $\varepsilon_{m n}$ sont respectivement les contraintes et déformations, $D^{\alpha_{k}}$ désigne l'opérateur de la dérivée fractionnaire au sens de Riemann-Liouville, défini précédemment, $\Gamma$ est la fonction Gamma (relation (2.4)), les paramètres $\alpha_{j}, \beta_{k}, \alpha_{l}$, et $\beta_{p}$ sont tous inférieurs à 1 , et $\lambda_{0}, \lambda_{j}, a_{k}, \mu_{0}, \mu_{1}$ et $b_{p}$ sont des constantes dépendant du matériau.

L'application de la transformée de Fourier à la relation (2.4), nous donne la relation suivante:

$$
\sigma_{m n}^{*}(\omega)=\delta_{m n} \lambda^{*}(\omega) \sum_{k} \varepsilon_{k k}^{*}(\omega)+2 \mu^{*}(\omega) \varepsilon_{m n}^{*}(\omega),
$$

où :

$$
\begin{array}{r}
\lambda^{*}(\omega)=\frac{\lambda_{0}+\sum_{j=1}^{J} \lambda_{j}(j \omega)^{\alpha_{j}}}{1+\sum_{k=1}^{K} a_{k}(j \omega)^{\beta_{k}}}, \\
\mu^{*}(\omega)=\frac{\mu_{0}+\sum_{l=1}^{L} \mu_{l}(j \omega)^{\alpha_{l}}}{1+\sum_{p=1}^{P} b_{p}(j \omega)^{\beta_{p}}},
\end{array}
$$

avec: $\sigma_{m n}^{*}(\omega)$ et $\varepsilon_{m n}^{*}(\omega)$ les transformées de Fourier de $\sigma_{m n}(t)$ et $\varepsilon_{m n}(t)$.

L'équation (2.4) est équivalente à l'intégrale de convolution:

$$
\sigma_{m n}(t)=\delta_{m n} \int_{-\infty}^{t} \lambda(t-\tau) \sum_{k} \dot{\varepsilon}_{k k}(\tau) d \tau+2 \int_{-\infty}^{t} \mu(t-\tau) \dot{\varepsilon}_{m n}(\tau) d \tau
$$

où

$$
\lambda(t)=\mathcal{F}^{-1}\left(\frac{\lambda^{*}(j \omega)}{j \omega}\right) \text { et } \mu(t)=\mathcal{F}^{-1}\left(\frac{\mu^{*}(j \omega)}{j \omega}\right)
$$

désignent les transformées de Fourier inverses.

Pour caractériser un matériau viscoélastique, on doit utiliser des essais dynamiques. Les essais vibratoires d'extension ou de torsion sur des tiges viscoélastiques sont les plus simples. Dans ce cas, l'utilisation d'un modèle monodimensionnel est 
adopté et la relation (2.4) donne la loi de comportement de Bagley - Torvik suivante:

$$
\sigma(t)+\sum_{k=1}^{K} a_{k} D^{\alpha_{k}}(\sigma(t))=E_{0} \varepsilon(t)+\sum_{l=1}^{L} b_{l} D^{\beta_{l}}(\varepsilon(t)),
$$

où : $\alpha_{k}$ et $\beta_{l}$ sont des valeurs fractionnaires comprises entre 0 et $1, \sigma(t)$ et $\varepsilon(t)$ sont respectivement les contraintes et déformations, $t$ est la variable temps, $a_{k}$ et $b_{l}$ sont des constantes caractéristiques.

La relation (2.10) a été utilisée dans les calculs en éléments finis faisant intervenir les élastomères dans le comportement dynamique des pneumatiques.

Seuls les premiers termes de chaque série de l'équation (2.10) sont pris en compte pour de nombreux matériaux. La loi de comportement s'écrit donc:

$$
\sigma(t)+a D^{\alpha}(\sigma(t))=E_{0} \varepsilon(t)+b D^{\beta}(\varepsilon(t)) .
$$

La transformée de Fourier de la relation (2.11) donne l'équation suivante:

$$
\left(1+a(j \omega)^{\alpha}\right) \sigma^{*}(\omega)=\left(E_{0}+b(j \omega)^{\beta}\right) \varepsilon^{*}(\omega),
$$

qui s'écrit encore sous la forme:

$$
\sigma^{*}(\omega)=E^{*}(\omega) \varepsilon^{*}(\omega)
$$

avec:

$$
E^{*}(\omega)=\frac{E_{0}+b(j \omega)^{\beta}}{1+a(j \omega)^{\alpha}}
$$

le module d'Young complexe.

Les relations (2.13)-(2.14) présentent un modèle à dérivées fractionnaires du module d'Young complexe des matériaux viscoélastiques. Cette forme a été utilisée par Bagley et Torvik [3], [2]. Les relations (2.13)-(2.14) sont équivalentes à la relation (2.2) utilisée par Vinh [22].

Pour un solide viscoélastique linéaire le module complexe à hautes fréquences doit être fini. On distingue deux possibilités : il faut

- soit introduire un facteur multiplicatif à la relation (2.14), ce qui donne la nouvelle forme:

$$
E^{*}(\omega)=\frac{E_{0}+b(j \omega)^{\beta}}{1+a(j \omega)^{\alpha}} \frac{1+a(j \omega)^{\alpha}}{1+a(j \omega)^{\beta}}, \quad \text { avec } \alpha \neq \beta,
$$

- soit prendre des valeurs égales pour $\alpha$ et $\beta$ et la relation (2.14) peut prendre la forme suivante:

$$
E^{*}(\omega)=\frac{E_{0}+b(j \omega)^{\alpha}}{1+a(j \omega)^{\alpha}} .
$$

Si nous posons

$$
a=\left(\frac{1}{\omega_{0}}\right)^{\alpha} \text { et } b=\left(\frac{1}{\omega_{1}}\right)^{\alpha}
$$

il vient:

$$
E^{*}(\omega)=\frac{E_{0}+\left(\frac{j \omega}{\omega_{1}}\right)^{\alpha}}{1+\left(\frac{j \omega}{\omega_{0}}\right)^{\alpha}} .
$$

Les expériences montrent que cette relation peut suffire pour décrire le comportement des polymères et élastomères, sauf dans le cas des matériaux comportant plusieurs zones de transitions différentes.

Généralement, les résultats expérimentaux se trouvent dans un intervalle faible de fréquences (2 à 4 décades) et l'utilisation d'un modèle à quatre paramètres rend 
l'étape d'identification de ces paramètres rhéologiques délicate. Pour faciliter cette étape, la nouvelle forme suivante du module complexe peut être utilisée [14], [15], $[16]$ :

$$
E^{*}(\omega)=\frac{E_{0}\left(1+\frac{1}{Z}\left(j \frac{\omega}{\omega_{0}}\right)^{\alpha}\right)}{1+\left(j \frac{\omega}{\omega_{0}}\right)^{\alpha}}
$$

où

$$
Z=\frac{E_{0}}{E_{\infty}}
$$

L'amortissement du matériau est donné par: $\eta=\frac{E_{2}(\omega)}{E_{1}(\omega)}$ (où l'on a décomposé $\left.E^{*}=E_{1}+j E_{2}\right)$ et l'amortissement maximum $\eta_{M}$ est donné par:

$$
\eta_{M}=\frac{E_{2}\left(\omega_{M}\right)}{E_{1}\left(\omega_{M}\right)}=\frac{(1-Z) Z^{1 / 2} \sin \left(\frac{\alpha \pi}{2}\right)}{2 Z+(1+Z) Z^{1 / 2} \cos \left(\frac{\alpha \pi}{2}\right)},
$$

ce qui donne:

$$
\alpha=\frac{2}{\pi} \arcsin \left[\frac{\eta_{M}\left[2(1-Z) Z^{1 / 2}+(1-Z)^{2}\left(1+\eta_{M}^{2}\right)^{1 / 2}\right]}{\eta_{M}^{2}(1+Z)^{2}(1-Z)^{2}}\right]
$$

où $\omega_{M}$ est la pulsation correspondant à l'amortissement maximum $\eta_{M}$. $\omega_{M}$ est lié à la variable adimensionnelle $Z$ par la relation $\omega_{M}=Z^{\alpha / 2}$. Ces formulations permettent de mettre en place un protocole d'estimation des paramètres du modèle.

\section{IDENTIFICATION DES PARAMÈTREs DU MODÈle À DÉRivÉEs FRACTIONNAIRE.}

La relation (2.18) représente le module complexe d'un matériau viscoélastique par un modèle à dérivées fractionnaires composé de quatre paramètres $E_{0}, Z, \alpha$ et $\omega_{0}$.

Le tracé du diagramme de Bode du module d'Young complexe fournit les valeurs asymptotiques $E_{0}$ et $E_{\infty}$, ainsi que la valeur de l'amortissement maximum $\eta_{M}$. La relation (2.19) nous donne immédiatement la valeur du paramètre $Z$ qui permet à son tour de déterminer $\alpha$ par la relation (2.21). Trois des quatre paramètres du modèle sont ainsi obtenus quasiment immédiatement à la lecture des relevés expérimentaux. La pulsation $\omega_{0}$, dernier paramètre du modèle, est obtenue en minimisant l'écart quadratique moyen entre le module d'Young expérimental et le module d'Young théorique. Ce processus d'optimisation conduit à une relation transcendante résolue par la méthode de Raphson-Newton [17]. La figure 1 motre l'organigramme d'identification des paramètres du modèle à dérivées fractionnaires.

ESAIM: Proc., VOL. 5, 1998, 193-204 


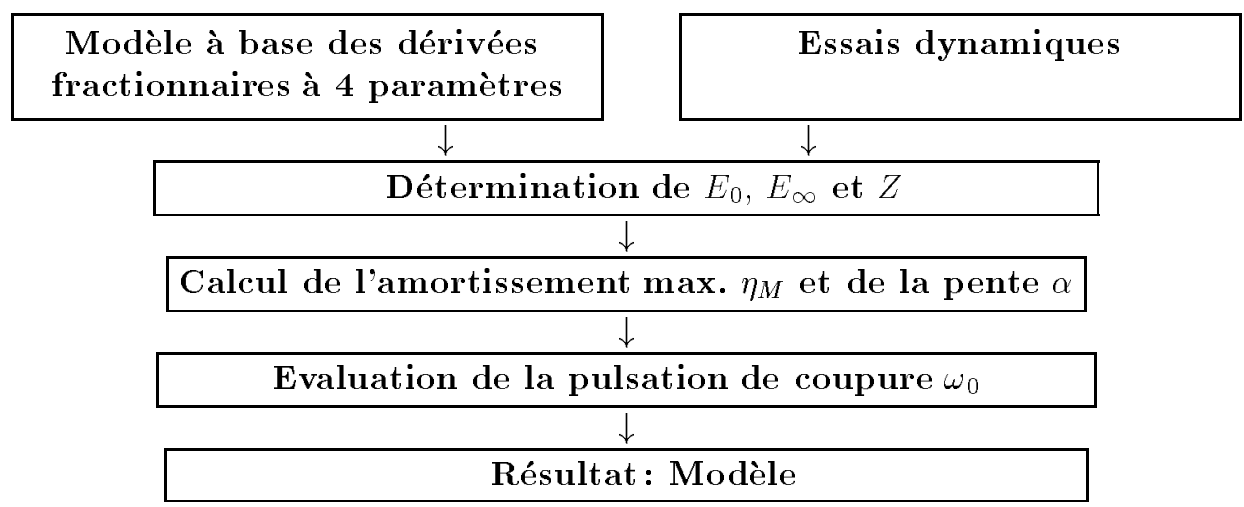

Fig. 1 -. Les différentes étapes de calcul des paramètres du modèle

\section{LOI DE COMPORTEMENT À BASE DES DÉRIVÉES FRACTIONNAIRES EN RÉGIME TRANSITOIRE.}

Ce problème a fait l'objet de nombreuses études en Rhéologie. De nombreux travaux, Alfrey [1], Tobolsky [20], Schwartz-Staverman [18], Ferry-Ninomiya [7] ont permis de passer d'un régime à l'autre.

Les préoccupations des rhéologues ne rejoignent pas forcément celles des mécaniciens. En effet les premiers veulent travailler sur des courbes dans une grande gamme de fréquence (plus de 10 décades fréquentielles) et à partir de ces dernières ils veulent obtenir les courbes temporelles dans une grande gamme de temps. On comprend aisément que les formules ci-dessus font intervenir des variables $\log (\omega)$ et $\log (\tau)$.

En mécanique, les préoccupations sont différentes. Dans un certain nombre d'applications on s'intéresse au domaine du temps court. Dans d'autres applications c'est l'inverse, c'est le domaine des temps longs qui est retenu [17].

Nous tentons dans cet article d'adopter le point de vue du mécanicien et nous présenterons deux développements asymptotiques différents selon le domaine temporel choisi.

Les temps courts sont utilisés dans les problèmes dynamiques rapides. Le développement asymptotique pour des temps courts (relaxation ou dynamique rapide) est obtenu par l'utilisation de la transformée inverse de Laplace ou le développement de Heaviside.

Les temps longs, dans les problèmes transitoires lents (fluage ou relaxation). Le développement asymptotique pour des temps longs (fluage) est obtenu en utilisant le contour de Bromwich-Wagner, ou de Sutton selon le cas.

Nous commençons par la méthode d'inversion directe de Madame Veysseyre [21], qui est une méthode exacte incluant comme cas particulier les deux développements asymptotique mentionnés.

\subsection{Méthode d’inversion directe de Mme Veysseyre}

Cette méthode est générale, elle permet la détermination de la fonction de relaxation à partir du module complexe en régime harmonique se trouvant dans une gamme de fréquences très étendue [22], [17]. 
Le module d'Young complexe (relation (2.18)) s'écrit:

$$
E^{*}(\omega)=\frac{E_{0}+E_{\infty}\left(j \frac{\omega}{\omega_{0}}\right)^{\alpha}}{1+\left(j \frac{\omega}{\omega_{0}}\right)^{\alpha}},
$$

ou encore:

$$
E^{*}(\omega)=\frac{E_{0}+E_{\infty}\left(T_{0} p\right)^{\alpha}}{1+\left(T_{0} p\right)^{\alpha}}, \quad \text { avec } p=j \omega \text { et } T_{0}=1 / \omega_{0} .
$$

L'utilisation de l'intégrale de Mellin-Fourier associée à un contour de BromwichWagner équivalent fig. 2 permet de calculer la réponse indicielle ou encore la réponse en régime transitoire $E(t)$, à partir de l'image de Carson:

$$
E(t)=\frac{1}{2 j \pi} \int_{c-j \infty}^{c+j \infty} e^{t p} \frac{E^{*}(p)}{p} d p .
$$

Il s'agit de calculer une fonction multiforme ayant un point de branchement à l'origine. Pour rendre la fonction analytique nous adoptons un contour de Bromwich muni d'une fente de $-\infty$ à 0 . Cette fonction est uniforme et holomorphe dans la région déterminée par le contour et sur le contour ce qui permet d'appliquer le théorème de Cauchy:

$$
\int_{C} g(z) d z=0,
$$

ce qui revient à évaluer (4.3) par l'intégrale (4.4) le long du contour figure 2, dans le sens de la flèche:

$$
E(t)=\frac{1}{2 j \pi}\left[\int_{\mathrm{C}_{1}}+\int_{\mathrm{DC}}+\int_{\mathrm{BA}}+\int_{\mathrm{C}_{2}}\right] .
$$

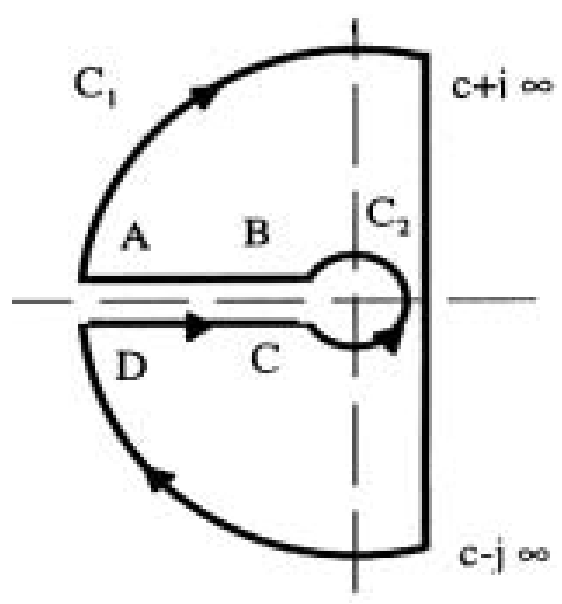

FIG. 2 -. Contour de Bromwich-Wagner

Quand le rayon de $\mathrm{C}_{1}$ tend vers $+\infty$, l'intégrale correspondante tend vers 0 . Pour le contour $\mathrm{C}_{2}$, l'intégrale tend vers 1 quand le rayon tend vers zéro. Il reste donc à calculer :

$$
E(t)=1+\frac{1}{2 j \pi}\left[\int_{\mathrm{DC}}+\int_{\mathrm{BA}}\right]
$$

sur le contour DC on pose

$$
p=r e^{-j \pi},
$$


sur le contour BA on pose

$$
p=r e^{+j \pi} \text {. }
$$

La fonction de relaxation $E(t)$ est alors donnée par la relation [14]:

$$
E(t)=E_{1}(t)+E_{2}(t)
$$

tel que:

$$
\begin{gathered}
E_{1}(t)=E_{0}\left\{e ^ { - \frac { t } { T _ { 0 } } } \sum _ { k = 0 } ^ { \infty } ( - 1 ) ^ { k \frac { \operatorname { s i n } ( k \pi \alpha ) } { k \pi \alpha } } \left[{ }_{1} F_{1}\left(1,1+\alpha k, \frac{t}{T_{0}}\right)+{ }_{1} F_{1}\left(1,1-\alpha k, \frac{t}{T_{0}}\right)\right.\right. \\
\left.\left.-e^{-\frac{t}{T_{0}}}\left(\frac{t}{T_{0}}\right)^{\alpha k} \Gamma(1-\alpha k)\right]\right\},
\end{gathered}
$$

et

$$
E_{2}=\mathcal{L}^{-1}\left[\frac{T_{0}^{\alpha}}{Z} p^{\alpha} E_{1}(p)\right]=\frac{T_{0}^{\alpha}}{Z} \frac{1}{\Gamma(-\alpha)} \int_{0}^{t}(t-\tau)^{-\alpha-1} E_{1}(\tau) d \tau
$$

4.2. Développement asymptotique de Heaviside pour des temps courts

Les temps courts correspondent à $|p|$ grand, soit $\left|T_{0} p\right| \gg 1$. L'expression du module (relation (4.2)) complexe dans ces conditions peut être approchée par l'expression suivante (à hautes fréquences) :

$$
E^{*}(p)=\frac{E_{\infty}\left(T_{0} p\right)^{\alpha}}{1+\left(T_{0} p\right)^{\alpha}}
$$

et le développement asymptotique de Heaviside pour des temps courts est donné par [14]:

$$
E(t)=E_{\infty}\left(\delta(t)+\sum_{i=1}^{\infty} \frac{(-1)^{i}}{T_{0}^{\alpha i}} \frac{t^{\alpha i-1}}{\Gamma(\alpha i)}\right)
$$

\subsection{Développement asymptotique de Sutton Pour Des temps longs}

Dans ce cas, nous supposons que $|p|$ soit tel que $\left|T_{0} p\right| \ll 1$. Le module complexe $E^{*}(p)$ prend la forme approchée suivante [14]:

$$
E^{*}(p)=\frac{E_{0}}{1+\left(T_{0} p\right)^{\alpha}},
$$

et le développement asymptotique de Sutton pour des temps longs est donnée par la relation [14]:

$$
E(t)=E_{0} \sum_{i=1}^{\infty}(-1)^{i} T_{0}^{\alpha i} \frac{t^{-\alpha i-1}}{\Gamma(-\alpha i)}
$$

\section{Application À Un ÉLAstomère}

\subsection{Modélisation DU MODUle COMPleXe Par les DÉrivéES FRACTIONNAIRES}

Les essais dynamiques de vibrations longitudinales sur des tiges d'un élastomère [15], [16] donnent le module complexe et l'amortissement de ce matériau en fonction des fréquences. La modélisation par les dérivées fractionnaires donne le modèle suivant:

$$
E^{*}(\omega)=\left(1.005 \frac{1+\frac{1}{3.23}\left(\frac{j \omega}{650}\right)^{0.65}}{1+\left(\frac{j \omega}{650}\right)^{0.65}}\right) \mathrm{GPa} .
$$

La figure 3 montre les résultats de la modélisation du module complexe d'un élastomère. 


\subsection{RÉPONSE EN RÉGIME TRANSITOIRE D'UN ÉLASTOMÈRE}

Reprenons l'élastomère modélisé en régime fréquentiel dans le paragraphe précédent 5.1. Le module d'Young de relaxation en fonction du temps est obtenu par la relation (4.9) dans laquelle les paramètres du modèle ont pour valeur $E_{0}=1 \mathrm{Gpa}$, $\alpha=0.65, \omega_{0}=650 \mathrm{~Hz}$ et $E_{\infty}=3,23 \mathrm{Gpa}$. La figure 4 montre une bonne corrélation entre le module d'Young de relaxation et ses estimations aux temps courts et aux temps longs données par (4.13) et (4.15) respectivement.

\section{Application au comportement viscó́lastique non linéaire}

Les élastomères, qu'ils soient compacts ou cellulaires, voire composites, présentent un comportement mécanique complexe. Pour de faibles niveaux d'efforts ces matériaux se déforment largement (de plusieurs centaines de \% parfois), les efforts internes sont de plus dépendants de la vitesse de sollicitation et de la température. Le comportement thermomécanique de tels matériaux doit être envisagé dans le cadre de la viscoélasticité non linéaire: non linéarités géométriques mais aussi non linéarités comportementales. Un certains nombre de modèles permettent de décrire le comportement non linéaire de matériaux, voir T. Béda, Y. Chevalier [4], les plus réalistes sur le plan de l'utilisation faisant appel à l'intégrale d'hérédité des milieux viscoélastiques. Dans bon nombre d'applications industrielles le matériau est soumis à une précharge statique définie par une élongation $\lambda_{0}$ autour de laquelle viennent se greffer des vibrations caractérisées par des petites déformations $\varepsilon(t)$. C'est le cas par exemple des plots d'amarrage en élastomères de structures vibrantes (machines tournantes, groupe moto-propulseur de véhicule, etc.). Dans le domaine fréquentiel, la loi de comportement est définie par une relation analogue à l'égalité (2.13) dans laquelle les paramètres $E_{0}, E_{\infty}, \omega_{0}$ et $\omega_{l}$ définissant le module d'Young complexe (relation (2.17) dépendent de l'élongation $\lambda_{0}$. Les essais sous précharge ne peuvent s'effectuer que dans une bande de fréquences très réduite $(0,200$ $\mathrm{Hz}$ dans le cas présent) mais à chaque élongation $\lambda_{0}$, une modélisation par dérivée fractionnaire permet d'extrapoler le comportement du matériau entre 0 et $10 \mathrm{kHz}$ environ. Le tableau 1 décrit l'évolution des paramètres du module d'Young complexe (relation (2.17)) d'un fluorosilicone, matériau fortement amortissant. Cette modélisation fait ressortir le comportement classique des élastomères: l'amplitude du module d'Young augmente avec la précharge tandis que l'amortissement diminue.

\begin{tabular}{|ccccc|}
\hline$\lambda_{0}$ & $E_{0}(\mathrm{Mpa})$ & $\alpha$ & $\omega_{0}(\mathrm{~Hz})$ & $\omega_{1}(\mathrm{~Hz})$ \\
& & & & \\
1 & 5.8 & 0.96 & 110 & 750 \\
0.8 & 8.0 & 0.75 & 35 & 800 \\
\hline
\end{tabular}

Tableau 1: Evolution de la modélisation par dérivée fractionnaire du module d'Young complexe d'un fluorosilicone

\section{CONCLUSION}

L'étude du comportement rhéologique des polymères dans le domaine de la reversibilité est gouverné par la viscoélasticité (linéaire ou non) dont le problème essentiel est la caractérisation mécanique de ces matériaux. Le problème reste complexe car ces lois constitutives dépendent du temps (ou de la fréquence) et de la température, si bien qu'aucun appareil ne peut fournir à lui seul le comportement 
dans une large plage de temps et une large gamme de température. L'expérimentateur a alors recours à divers artifices tels que l'utilisation de plusieurs appareillages ou la superposition temps-températures. La dérivée fractionnaire, qui autorise le passage continu de l'état de "ressort" à celui "d'amortisseur", est un outil précieux de modélisation en mécanique car il permet, à précision égale, de minimiser le nombre de paramètres du modèle.

Cette modélisation, qui rencontre quelques réticences au niveau des utilisateurs de logiciels de calcul de structures par éléments finis (lourdeur des expressions temporelles) ouvre cependant des perspectives intéressantes dans l'étude des comportements rhélogiques non linéaires des matériaux.

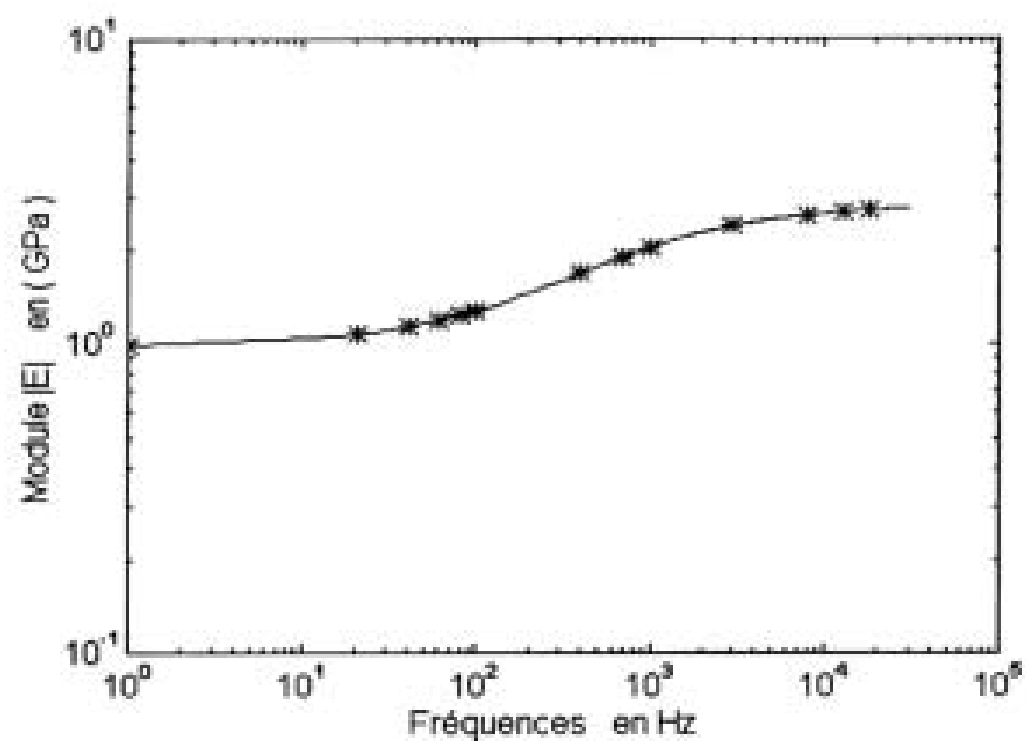

Fig. 3 -. Module complexe d'un élastomère - ***: résultats expérimentaux, -: modèle à dérivées fractionnaires (essai de tractioncompression).

\section{RÉFÉRENCES}

[1] T. ALFREY et P. DOTY, J. Appl. Phys., 16, pp. 700-, (1945).

[2] R. L. BAGLEY and P. J. TORVIK, "A Theoretical Basis for The Application of Fractional Calculus to Viscoelasticity". Journal of Rheology, 27(3), pp. 201-372, (1983).

[3] R. L. BAGLEY and P. J. TORVIK, "On The Fractional Calculus Model of Viscoelastic Behavior". Journal of Rheology, 30(1), pp. 133-155, (1986).

[4] T. BEDA, Y. CHEVALIER, "Sur le comportement statique et dynamique des élastomères en grandes déformations", Mécanique industrielle et matériaux, vol. 50, n. 5, pp. 228-231, (1997).

[5] M. CAPUTO et F. MAINARDI, "A New dissipation Model Based on Memory Mechanism", Pure and Applied Geophysics, vol. 91, pp. 134-147, (1971).

[6] S. DUGOWSON, "L'élaboration par Riemann d'une définition de la dérivation d'ordre non entier", Revue d'histoire des mathématiques, 3, pp. 49-97, (1997).

[7] J. D. FERRY, "Viscoelastic property of polymers" 2nd edition. John Wiley Edit, New York, (1970).

[8] A. K. GRÜNWALD, "Dérivationen und deren Anwendung", Zeitschrift für Mathematik und Physik, 12, pp. 441-480, (1867).

[9] G. W. LEIBNIZ, "Letter from Hanover, Germany, September 30, 1695 to G. A. L'Hospital". Leibnizen Mathematische Schriften, vol. 2, pp. 301-302. Olms Verlag, Hildesheim, Germany, (1962). First published in 1849. 


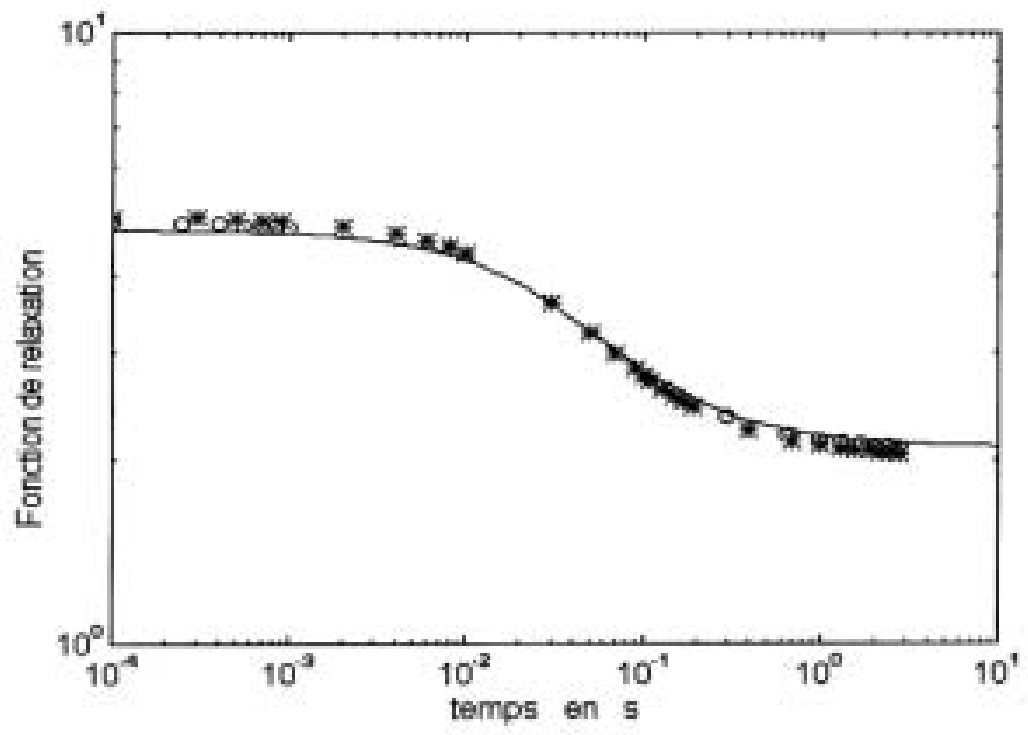

Fig. 4 -. Fonction de relaxation d'un élastomère - ***: méthode d'inversion directe, ooo: développements asymptotiques en temps longs et en temps courts.

[10] J. LIOUVILLE, "Mémoire sur le calcul des différentielles à indices quelconques", J. Ecole polytechnique. 13, n. 221 , pp. $71-162,(1832)$.

[11] P. G. NUTTING, J. Franklin Inst., 191, pp. 679-685, (1921).

[12] P. E. ROUSE, "A theory of the linear viscoelastic properties of dilute Solutions of coiling polymers", The Journal of Chemical Physics, vol. 21, n. 7, (1953).

[13] W. SMIT and H. VERIES, "Rheological models containing fractional derivatives", Rheol. Acta 9, pp. 525-534, (1970).

[14] M. SOULA, "Etude du comportement mécanique des matériaux viscoélastiques par les dérivées fractionnaires", Thèse de Doctorat, CNAM, Paris, (1996).

[15] M. SOULA , T. VINH et Y. CHEVALIER, "Etude dynamique des polymères et élastomères par les dérivées fractionnaires", Mécanique industrielle et matériaux, vol. 49, n. 2, pp. 101103, (1996).

[16] M. SOULA , T. VINH et Y. CHEVALIER, "Measurements of isothermal complex moduli of viscoelastic matérials over a large range of frequencies" J. Sound and Vibration, 205(2), pp. $167-184,(1997)$.

[17] M. SOULA, T. VINH, Y. CHEVALIER et al., "Transient responses of polymers and elstomers deduced from harmonic responses", J. Sound and Vibration, 205(2), pp. 185-203, (1997).

[18] A. J. STAVERMAN and SCHWARZL, in H. A. Atuart, "Die Physik der Hochpolymern", vol. IV, chapter 1, Springer-Verlag, (1956).

[19] THIRION-CHASSET, "Constributions relatives de la viscoélasticité et du vieillissement à la relaxation du caoutchouc", Revue gén. Caout. et des plastiques. n. 41, pp. 271-276, (1949).

[20] TOBOLSKY - AKLONIS, "Relation between creep and stress relaxation", J. appl. phys. americ., vol. 37, n. 5, pp. 1949-, (1952).

[21] R. VEYSSEYRE, T. VINH, "Sur le passage du régime harmonique au régime transitoire en viscoélasticité", C. R. Académie des Sciences t. 266, Série A, (1968).

[22] T. VINH, "Sur le Passage du Régime Harmonique au Régime Transitoire viscoélastique", Extrait du mémorial de l'Artillerie Francaise, 3e Fasc, pp. 725-776, (1967). 